\title{
ESTIMATION OF PATIENT-SPECIFIC MATERIAL PROPERTIES OF THE MITRAL VALVE USING 4D TRANSESOPHAGEAL ECHOCARDIOGRAPHY
}

\author{
Jingjing Kanik ${ }^{1}$, Tommaso Mansi ${ }^{2}$, Ingmar Voigt ${ }^{2}$, Puneet Sharma ${ }^{2}$, Razvan Ioan Ionasec ${ }^{2}$, \\ Dorin Comaniciu ${ }^{2}$ and James Duncan ${ }^{1,3,4}$ \\ Department of ${ }^{1}$ Biomedical Engineering, ${ }^{3}$ Electrical Engineering, ${ }^{4}$ Diagnostic Radiology, \\ Yale University, New Haven, CT, USA \\ ${ }^{2}$ Siemens Corporation, Corporate Technology, Imaging and Computer Vision, Princeton, NJ, USA
}

\begin{abstract}
4D Transesophageal Echocardiography (TEE) is a newly developed tool to visualize the morphology and dynamics of the mitral valve for diagnosis and treatment planning. Quantitative patient-specific modeling of the mitral valve is demanded since it allows for the reliable predictive simulation of medical intervention. State-of-the-art image-based and biomechanical models with generic material parameters have limited predictive power as they are only partially fitted to patient-data. As a step closer to a fully personalized model, an estimation algorithm is presented in this paper. The method combines image-derived mitral valve dynamics with a biomechanical model to estimate regional patient-specific material parameters in-vivo. In particular, the extended Kalman filter (EKF) is adopted in a way that it becomes flexible to integrate any biomechanical model and more parameters of interest in the estimation. The algorithm was verified on synthetic data with known Young's modulus and shear modulus, yielding less than 5\% error. The algorithm was also evaluated on 4D TEE images of five patients. Estimated Young's moduli agree with the clinical observation that material parameters vary regionally and among the population. The estimated material parameters can be used in patient-specific modeling as well as the detection and evaluation of diseased areas.
\end{abstract}

Index Terms-TEE, mitral valve, biomechanical model, material parameters, extended Kalman filter

\section{INTRODUCTION}

Transesophageal Echocardiography (TEE) uses a special probe in the esophagus to obtain images of cardiovascular structures. TEE offers clearer images of the cardiac morphology compared to traditional echocardiography since the signal is not obstructed by ribs, obesity or emphysema. Newly developed 4D TEE provides a fast and easy tool for advanced visualization of the mitral valve. The mitral valve regulates blood flow from the left atrium to the ventricle through rapid opening and closing. Mitral valve dysfunction, including mitral stenosis and regurgitation where the valve cannot open or close properly, causes cardiac inefficiency or even lifethreatening conditions. Surgical intervention is necessary to repair or replace the diseased valve. A detailed preoperative plan is essential to choose a suitable treatment strategy and bring an optimized outcome [1]. Quantitative patient-specific modeling of the mitral valve is a challenging problem but can improve understanding of mitral valve function and assist clinicians during treatment planning.

Several approaches have been proposed to model mitral valve geometry and dynamics, including image-based and biomechanical methods. The image-based methods focus on estimating the mitral apparatus and dynamics from medical images. Schneider et al. [2] proposed a semi-automatic method to segment and track mitral valve annulus and leaflets during the cardiac cycle from 4D ultrasound images. Ionasec et al. [3] proposed a learning based fully automatic method that extracts the mitral valve dynamics from 4D cadiac computed tomography or TEE. These methods can provide accurate morphological and functional quantification of the mitral valve but do not explain the underlying mechanisms of the pathology. Voigt et al. [4] proposed a similar learning based method but incorporated the leaflets' biomechanics to ensure temporal and physiological consistency. However, those methods above do not use personalized material properties therefore lack predictive power in guiding the preoperative treatment plan. In the last decade, several computational mechanical models of the mitral valve have been proposed since the pioneering work of Kunzelman et al. [5]. Patient-specific mechanical models are built from medical images $[6,7]$ where the mitral apparatus is estimated from the images while the material parameters of the mitral leaflet tissues are obtained from experimental results and generalized to all the patients. However, studies on sheep data [8] show that the material properties vary both among individuals as well as regionally. Material properties vary among patients especially in the diseased area due to pathological changes. Hence personalized material parameters are important to accurately model the mitral valve and predicatively plan appropriate therapy.

In this paper an estimation framework is presented com- 


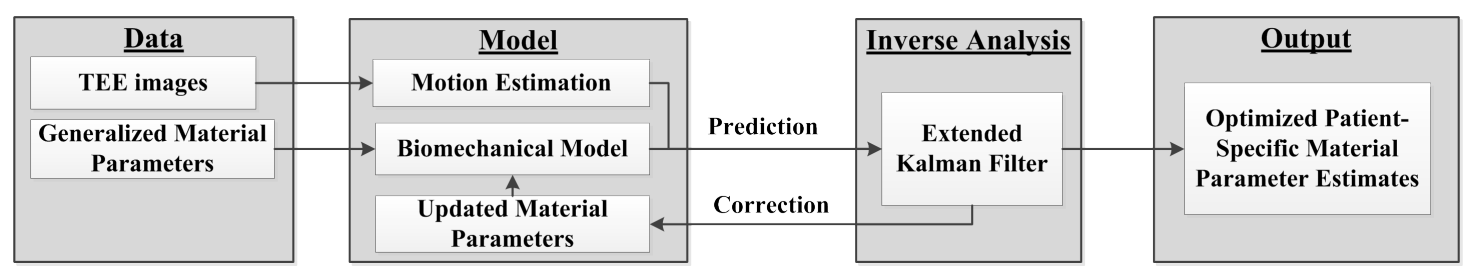

Fig. 1. Proposed framework for the mitral valve material parameter estimation

bining the image-based and biomechanical models to estimate patient-specific material parameters of the mitral valve. It is the first study, to the best of our knowledge, to estimate invivo material parameters of mitral leaflets based on the observed motion pattern from TEE. The method was tested on synthetic data and five patients with promising accuracy.

\section{METHOD}

\subsection{Overview of the method}

A two step procedure is followed for the proposed method as shown in Figure 1: First, the mitral valve geometry sequence is estimated from TEE images. Then, the motion sequence is treated as an observation of the outcome of the mitral valve system and the estimation is performed to fit the image-based observation into the biomechanical model to estimate patientspecific material parameters. The details of each step are explained in the next two subsections. The extended Kalman filter (EKF) approach, which was proposed by Shi et al. [9] in $2 \mathrm{D}$ left ventricle motion analysis, is employed for the estimation. The EKF is chosen since it provides a stable and efficient sequential least square solution to periodic mitral valve dynamics. The standard EKF framework is adapted in way that allows the mechanical model to be kept as a relatively independent module so that it is easy to incorporate future more complex mechanical models.

\subsection{Image-based and biomechanical model}

The important anatomical components of the mitral valve are the mitral annulus, the anterior and posterior leaflets, papillary tips and chordae. The 4D TEE images are processed using the hierarchical discriminative learning algorithm [3] to extract the geometry and corresponding motion. The triangulated surface mesh (Fig 2.(a)) for the leaflets and two papillary tips are generated from the images and the algorithm ensures intra- and inter-patient point correspondence of vertices. The mitral annulus and papillary tip motion is also quantified from the images to be used as boundary condition in the biomechanical model. The leaflet thickness is set to be $1.32 \mathrm{~mm}$ and $1.26 \mathrm{~mm}$ for the anterior and posterior leaflet respectively [7] to construct the volumetric structure. Finally, tetrahedral meshes (Fig2.(b)) are generated from the volumetric structures for the biomechanical model. The marginal and
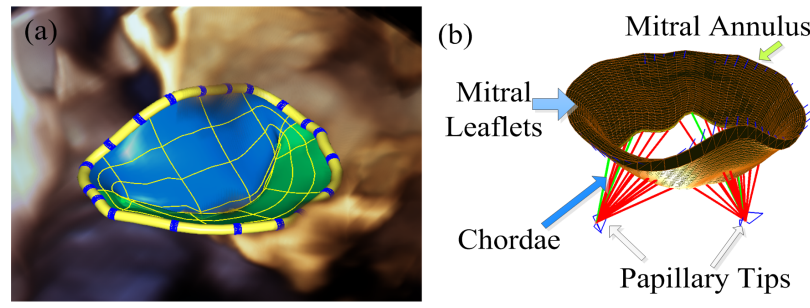

Fig. 2. The mitral apparatus represented by (a) Triangulated meshes (b) Tetrahedral meshes

basal chordae are attached between papillary tips and leaflets as in [7]. In particular, the insertion points are determined by visual inspection and identical for all patients thanks to the point correspondence.

A finite element method is used to simulate mitral valve biomechanics implemented in SOFA ${ }^{1}$. The leaflets are modeled as linear, transverse isotropic and nearly incompressive tissues. Leaflet collagen fibers are modeled as in [7], where they are mainly parallel to the annulus while those in anterior leaflet close to the commissures gradually rotate to become perpendicular to the annulus. The mitral valve is governed by the system dynamics:

$$
M \ddot{U}+C \dot{U}+K U=F
$$

where $\mathrm{U}$ is the displacement vector of the vertices of the mitral valve mesh, $\dot{U}$ and $\ddot{U}$ are the velocity and acceleration respectively. $\mathrm{M}$ is the diagonal mass matrix (a uniform mass density $\rho=1.04 \mathrm{~g} / \mathrm{ml}$ is used), $\mathrm{K}$ is the stiffness matrix and a function of material parameters, and $\mathrm{C}$ is the Raleigh damping matrix $(\mathrm{C}=0.1(\mathrm{M}+\mathrm{K}))$. $\mathrm{F}$ is the total force developed by the chordae and heart pressure. Mitral leaflet material proporties are the focus of this study therefore generic pressure profile and chordae forces are applied. However, it is noted that chordae forces, which are related to the material properties, morphology and elongation of the chordae, have a significant influence on mitral valve dynamics.

\subsection{Estimation framework}

The material parameters of interest in this study include Young's modulus $(E)$ and shear modulus $(G)$ along and across the leaflet collagen fibers ( $E_{A L_{f}}, E_{A L_{f_{\perp}}}, G_{A L}, E_{P L_{f}}$,

\footnotetext{
${ }^{1}$ http://www.sofa-framework.org/
} 
$E_{P L_{f_{\perp}}}$ and $G_{P L}$ for the anterior and posterior leaflets respectively). Poisson ratio $(\nu)$ is set to be 0.488 due to the incompressive nature of the tissues. The ratio of Young's modulus along and across the fiber $\left(r=\frac{E_{f}}{E_{f \perp}}\right)$ is fixed and the shear modulus is approximated by $G \approx E_{f} /(2(1+\nu))$ to ensure the physiological consistency in the estimation.

The dynamic equilibrium equation can be transformed into a state space representation of the system and the system can be solved by the EKF. The state vector $\mathbf{m}_{\mathbf{k}}=$ $\left[m_{1 k}^{1}, m_{2 k}^{1}, \ldots m_{J k}^{1}, \ldots, m_{1 k}^{I}, m_{2 k}^{I}, \ldots m_{J k}^{I}\right]$ is the vector of $\mathbf{J}$ number of the material parameters in I number of different regions at the $k_{t h}$ frame. In this study $\mathbf{m}_{\mathbf{k}}=\left[E_{A L_{f} k}, E_{P L_{f} k}\right]=$ $\left[m_{k}^{1}, m_{k}^{2}\right]$ is the target of estimation and the other four parameters $\left(E_{A L_{f_{\perp}}}, G_{A L}, E_{P L_{f_{\perp}}}, G_{P L}\right)$ can be derived from $\mathbf{m}_{k}$. The process function $f(\cdot)$ is derived from the assumption that material parameters stay constant during the cardiac cycle. The observation vector $\mathbf{g}_{\mathbf{k}}=\left[x_{k 1}, y_{k 1}, z_{k 1}, \ldots, x_{k i}, y_{k i}, z_{k i}, \ldots\right.$, $\left.x_{k L}, y_{k L}, z_{k L}\right]$ is the geometry vector which is represented by $\mathrm{L}$ number of vertices ( $\mathrm{L}=3248$ in this study). The observation function $h(\cdot)$ is derived from the specific biomechanical model. The model described in section 2.2 is used in this study. The formulation keeps the finite element model as an independent module in the observation equation. The state space representation is as follows:

$$
\begin{gathered}
\mathbf{m}_{k}=f\left(\mathbf{m}_{k-1}\right)+\mathbf{w}_{k-1}=\mathbf{m}_{\mathbf{k - 1}}+\mathbf{w}_{k-1} \\
\mathbf{g}_{\mathbf{k}}=h\left(\mathbf{m}_{k}\right)+v_{k}
\end{gathered}
$$

where $w_{k-1}$ and $v_{k}$ are the state and process noises respectively and assumed to follow Gaussian distributions with covariance matrix $Q_{k-1}$ and $R_{k}$. The estimation is initialized by setting the value of material parameters $\left(\mathbf{m}_{0}\right)$ and its covariance matrix $\left(P_{0}\right)$. The initial material parameters and covariance matrix are set to be the generic material parameters and diagonal matrix $\left(P_{0}=0.01 I\right)$ in this study. The EKF follows a prediction-correction process. In the prediction step, the material parameters $\mathbf{m}_{\mathbf{k}}^{\mathbf{f}}$ are predicted to be the same as last estimates. In the correction step, the predicted material parameters $\mathbf{m}_{\mathbf{k}}^{\mathbf{f}}$ are used in the biomechanical model to predict the geometry at $\mathrm{k}$, and then the predicted geometry $h\left(\mathbf{m}_{\mathbf{k}}^{\mathbf{f}}\right)$ is compared to the observation $\mathbf{g}_{\mathbf{k}}$ to generate new estimates $\mathbf{m}_{\mathbf{k}}^{\mathbf{a}}$. The recursive filtering procedure is described in equations as follows:

Prediction: $\quad \mathbf{m}_{\mathbf{k}}^{\mathbf{f}} \approx f\left(\mathbf{m}_{k-1}^{a}\right)=\mathbf{m}_{\mathbf{k}-\mathbf{1}}^{\mathbf{a}}$

$$
P_{k}^{f} \approx J_{f}\left(\mathbf{m}_{k-1}^{a}\right) P_{k-1} J_{f}^{T}\left(\mathbf{m}_{\mathbf{k}-\mathbf{1}}^{\mathbf{a}}\right)+Q_{k-1}=P_{k-1}+Q_{k-1}
$$$$
\text { Correction: } \quad \mathbf{m}_{k}^{a}=\mathbf{m}_{\mathbf{k}}^{\mathbf{f}}+K_{k}\left(\mathbf{g}_{\mathbf{k}}-h\left(\mathbf{m}_{\mathbf{k}}^{\mathbf{f}}\right)\right)
$$

$$
\begin{gathered}
K_{k}=P_{k}^{f} J_{h}^{T}\left(\mathbf{m}_{k}^{f}\right)\left(J_{h}\left(\mathbf{m}_{\mathbf{k}}^{\mathbf{f}}\right) P_{k}^{f} J_{h}^{T}\left(\mathbf{m}_{\mathbf{k}}^{\mathbf{f}}\right)+R_{k}\right)^{-1} \\
P_{k}=\left(I-K_{k} J_{h}\left(\mathbf{m}_{\mathbf{k}}^{\mathbf{f}}\right)\right) P_{k}^{f}
\end{gathered}
$$

where $J_{f}$ and $J_{h}$ are the Jacobian matrices of $f(\cdot)$ and $h(\cdot)$. $J_{f}$ is an identity matrix $I$ in this study. $J_{h}$ can be calculated from the finite element model outside the main flow. The iterative process is stopped when there is little improvement between the two consecutive iterations.

\begin{tabular}{|c|c|c|c|c|c|c|}
\hline & $E_{A L_{f}}$ & $E_{A L_{f}}$ & $G_{A L}$ & $E_{P L_{f}}$ & $E_{P L_{f}}$ & $G_{P L}$ \\
\hline True (MPa) & 6.23 & 2.35 & 2.09 & 2.09 & 1.89 & 0.70 \\
\hline $\begin{array}{c}\text { Estimation } \\
\text { (MPa) }\end{array}$ & 6.02 & 2.27 & 2.02 & 2.13 & 1.93 & 0.72 \\
\hline Error (\%) & 3.37 & 3.40 & 3.35 & 1.91 & 2.12 & 2.86 \\
\hline
\end{tabular}

Table 1. Results on the synthetic data

\begin{tabular}{|c|c|c|c|c|c|c|}
\hline & $E_{A L_{f}}$ & $E_{A L_{f}}$ & $G_{A L}$ & $E_{P L f}$ & $E_{P L_{f}}$ & $G_{P L}$ \\
\hline P1 & 6.28 & 2.38 & 2.11 & 2.61 & 2.36 & 0.88 \\
\hline P2 & 6.63 & 2.50 & 2.23 & 1.60 & 1.44 & 0.54 \\
\hline P3 & 5.62 & 2.12 & 1.89 & 3.03 & 2.74 & 1.02 \\
\hline P4 & 4.12 & 1.55 & 1.38 & 3.81 & 3.45 & 1.28 \\
\hline P5 & 5.73 & 2.16 & 1.92 & 2.87 & 2.59 & 0.96 \\
\hline
\end{tabular}

Table 2. Estimated material parameters of the patients

\section{EXPERIMENTAL RESULTS}

\subsection{Verification on synthetic data}

The proposed method was evaluated on synthetic dynamic motion. The synthetic motion was generated from the biomechnical model using the mitral valve geometry at the open state from one patient and known material parameters (the first row of Table 1) with time interval $t=0.01 \mathrm{~s}$. Only two frames, the initial and final frame when mitral valve is at open and closed state, were chosen as the observations since the mitral closure is the primary goal of the study. The estimation is initialized by $\mathbf{m}_{\mathbf{0}}=[\mathbf{5}, \mathbf{3}]$ and $P_{0}=0.01 I$. The covariance matrices $\left(R_{k}, Q_{k}\right)$ were all set to be 0.01 times identity matrices. The estimated material parameters were compared to the ground truth shown in Table 1 . The mean estimation error is $2.84 \pm 0.67 \%$ which is quite small. The Euclidean distance between the estimated and observed gemetries, which was calculated from the average pointwise euclidean distances because of the point correspondence among the meshes, is $0.06 \pm 0.07 \mathrm{~mm}$.

\subsection{Evaluation on the patients}

The presented method was also tested on five patients with TEE images. The mitral apparatus was automatically extracted from TEE images using the method described in 2.2. Similar to 3.1, the geometries at open and closed states derived from TEE were used as the observations for the estimation. The estimation is initialized by the general material parameters (shown in the first row of Table 1) and $P_{0}=0.01 I$. The covariance matrices $\left(R_{k}, Q_{k}\right)$ were all set to be 0.01 times identity matrices. The optimized patientspecific material parameters are estimated by minimizing the difference between the image derived and mechanical model derived mitral valve closure through EKF. However, it is difficult to validate the true in-vivo material properties for each 


\begin{tabular}{|l|c|c|c|c|c|}
\hline & $\begin{array}{c}\text { P1 } \\
(\mathrm{mm})\end{array}$ & $\begin{array}{c}\text { P2 } \\
(\mathrm{mm})\end{array}$ & $\begin{array}{c}\text { P3 } \\
(\mathrm{mm})\end{array}$ & $\begin{array}{c}\text { P4 } \\
(\mathrm{mm})\end{array}$ & $\begin{array}{c}\text { P5 } \\
(\mathrm{mm})\end{array}$ \\
\hline Generalized & $1.50 \pm$ & $2.65 \pm$ & $2.17 \pm$ & $1.94 \pm$ & $2.43 \pm$ \\
Parameters & 0.89 & 1.48 & 1.32 & 1.37 & 1.39 \\
\hline Personalized & $1.47 \pm$ & $2.25 \pm$ & $1.91 \pm$ & $1.74 \pm$ & $2.27 \pm$ \\
Parameters & 0.89 & 1.27 & 1.18 & 1.34 & 1.40 \\
\hline
\end{tabular}

Table 3. The Euclidean distances between the mitral valve closure generated from the biomechanical model and TEE
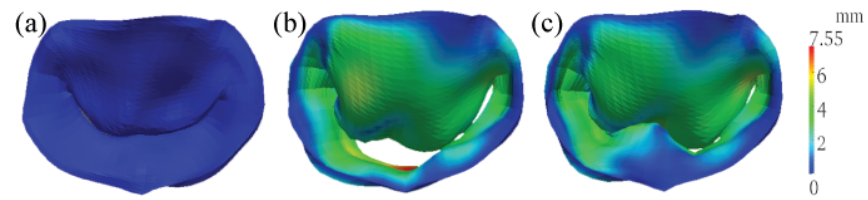

Fig. 3. (a) Mitral valve closure derived from TEE of Patient 2; The colored pointwise Euclidean distances to (a) from biomechanical model with (b) general material parameters; (c) estimated material parameters

patient since there is no direct measurement in clinical data.

The estimated material parameters (Table 2) show that anterior leaflet is stiffer than posterior leaflet in all patients which matches the experimental observations $[5,8]$. The results also suggest that material parameters vary among the patients thus further proving that it is necessary to tailor the material parameters to handle inter-patient complications in predictive modeling. The Euclidean distances between the image derived and mechanical model derived geometries are shown in Table 3. The personalized material parameters yield smaller distances, indicating that they model the mitral valve more closely. Greater improvement is expected by incorporating the chardae biomechanical parameters and resting length in future estimation, which are known to have a significant influence on the results [7]. The colored pointwise distances for Patient 2 (Fig. 3) show that the personalized parameters result in better closure of the mitral valve.

\section{CONCLUSION AND FUTURE WORK}

A novel algorithm for the analysis of the mitral valve dynamics is presented to estimate patient-specific material parameters. The quantitative evaluation on synthetic data demonstrates that the presented method works efficiently with less than 5\% estimation error. The results from the patients' data show that the material parameters are regionally and individually different. The presented algorithm was tested on TEE data but its application can be extended to cardiac Computed Tomography (CT) and Magnetic Resonance (MR) images. Future directions will include the estimation of material parameters of the chordae and utilizing the observed motion from the whole cardiac cycle in the estimation.

\section{REFERENCES}

[1] T. Feldman, S. Kar, M. Rinaldi, P. Fail, J. Hermiller, R. Smalling, P. Whitlow, W. Gray, R. Low, H. Herrmann, "Percutaneous mitral repair with the mitraclip system: safety and midterm durability in the initial everest (endovascular valve edge-to-edge repair study) cohort," Journal of the American College of Cardiology, 2009, 54, 686-694.

[2] R. Schneider, N. Tenenholtz, D. Perrin, G. Marx, P. del Nido, and R. Howe, "Patient-specific mitral leaflet segmentation from 4D ultrasound," Medical Image Computing and Computer-Assisted Intervention-MICCAI, 2011, pp. 520-527.

[3] R.I. Ionasec, I. Voigt, B. Georgescu, Y. Wang, H. Houle, F. Vega-Higuera, N. Navab, and D. Comaniciu, "Patientspecific modeling and quantification of the aortic and mitral valves from 4-D cardiac CT and TEE," IEEE Trans. Med. Imaging, 2010, 29(9): 1636-1651

[4] I. Voigt, T. Mansi, R. Ionasec, E. Mengue, H. Houle, B. Georgescu, J. Hornegger, and D. Comaniciu, "Robust physically-constrained modeling of the mitral valve and subvalvular apparatus," Medical Image Computing and Computer- Assisted Intervention-MICCAI 2011, pp. 504-511.

[5] K. Kunzelman, R. Cochran, C. Chuong, W. Ring, E. Verrier, and R. Eberhart, "Finite element analysis of the mitral valve," The Journal of heart valve disease, 1993, 2: 326-340.

[6] Q. Wang and W. Sun, "Finite element modeling of mitral valve dynamic deformation using patient-specific multislices computed tomography scans," Ann Biomed Eng, 2012 Jul 18.

[7] T. Mansi, I. Voigt, B. Georgescu, X. Zheng, E.A. Mengue, M. Hackl, R.I. Ionasec, T. Noack, J. Seeburger, and D. Comaniciu, "An integrated framework for finiteelement modeling of mitral valve biomechanics from medical images: application to MitralClip intervention planning," Med Image Anal. 2012 Oct;16(7):1330-46

[8] G. Krishnamurthy, A. Itoh, W. Bothe, J. Swanson, E. Kuhl, M. Karlsson, D. Craig Miller, and N. Ingels, "Stress-strain behavior of mitral valve leaflets in the beating ovine heart," Journal of biomechanics, 2009:42, 1909-1916.

[9] P. Shi and H. Liu, "Stochastic finite element framework for simultaneous estimation of cardiac kinematic functions and material parameters," Med Image Anal. 2003;17(4):445-464 